Results In 6 focus groups including 36 rheumatologists (37\% male), the key dilemma in lupus pregnancy was reported to be medically ill-timed pregnancies. We identified four modifiable barriers to effective pregnancy planning: 1.) physician/patient communication about contraceptive use was often directive, which is ill-suited to the sensitive discussion; 2.) some rheumatologists have a knowledge gap, with only $37 \%$ identifying mycophenolate mofetil as a major teratogen and most greatly over-estimating the efficacy of Depo-provera ${ }^{\circledR} ; 3$.) local rheumatologists and $\mathrm{OB} / \mathrm{GYN}$ frequently did not communicate, leading to varying treatment plans and confusion for patients; and 4.) pregnancy planning conversations were derailed by limited patient-trust and physician-empathy about a young womans desire to be a mother.

Conclusions While we hope that the coming inaugural ACR Reproductive Health Guidelines will improve pregnancy management and outcomes for women with SLE, prior research demonstrates that simple guideline publication does little to influence care. We have identified several important, modifiable factors that, if addressed, may allow the guidelines to have a stronger impact on pregnancy success. We have built HOP-STEP (Healthy Outcomes in Pregnancy in women with SLE Through Educating Providers), an in-person and online program, to enable open and honest conversations about pregnancy planning and management between rheumatologists and women with lupus (www.LupusPregnancy.org).

Funding Source(s): AHRQ: K18HS023443

\section{PREGNANCY INTENTION AMONG WOMEN WITH SLE AND OTHER RHEUMATIC DISEASES}

Megan EB Clowse*. Duke University

10.1136/lupus-2019-|sm.106

Background An estimated half of all pregnancies in the US are unplanned. In general, unplanned pregnancies are at higher risk for pregnancy loss and birth defects; an estimated $1 / 3$ are electively terminated. Among women with rheumatic disease, the risks of unplanned pregnancy are even higher due to the frequent use of teratogenic medications and complications from active disease at conception. We sought to determine the frequency of unplanned pregnancy and the demographic differences between planned and unplanned pregnancies in a university-based pregnancy and rheumatology clinic.

Methods At enrollment into a prospective rheumatology pregnancy cohort, each woman completed the London Measure of Unplanned Pregnancy (LMUP), a validated 6question survey that assesses a womans intentions for conception. Demographic and historical rheumatologic data were collected. A higher LMUP score indicated a higher degree of pregnancy planning; a planned pregnancy has a score of $10-12$.

Results Eighty-two pregnant women completed the survey between January and December 2018. The majority of women were white (68\%), well-educated $(77 \%$ at least college educated), married (77\%), and had private insurance $(77 \%)$; the average age was 30.7 . The most common diagnosis was SLE (26\%), followed by RA (21\%) and UCTD (18\%).
The median LMUP score was 11 with $71 \%$ of women having a planned pregnancy. Of the women with an unplanned pregnancy, only $37 \%$ reported contraceptive use, including progesterone-only pill (8\%), IUD (4\%), and barrier methods (25\%). No differences in contraception use between women with and without SLE were observed.

In comparison to women without SLE, those with SLE were more likely to have an unplanned pregnancy (25\% without SLE vs $43 \%$ with SLE, $\mathrm{p}=0.2$ ) The LMUP score was significantly lower for women with SLE (mean 10.3 non-SLE vs 9.0 SLE, $p=0.07$; median 12.0 non-SLE vs 10.0 SLE, $\mathrm{p}=0.01$ ). Only $43 \%$ of women with SLE reported that they had intended to get pregnant, compared to $75 \%$ of non-SLE women $(\mathrm{p}=0.02)$ and $48 \%$ of women with SLE reported taking folic acid or a prenatal vitamin compared to $72 \%$ of nonSLE women $(\mathrm{p}=0.06)$

Conclusions Among this largely upper-middle and middle class population, most pregnancies were planned and most women took steps pre-pregnancy to prepare; this data may not reflect the typical rheumatology clinic. Among women with SLE, however, a higher proportion of pregnancies were unplanned. Our next steps will be to correlate pregnancy intention with outcomes and implement approaches to limit unplanned pregnancies among women with SLE.

Funding Source(s): None

\section{FEATURES OF NEUROPSYCHIATRIC LUPUS IN NBA2 MOUSE MODEL}

${ }^{1}$ Emily Zhang*, 'Kirsten Evonuk, ${ }^{2} J e s s i c a$ Liu, ${ }^{1}$ Tara DeSilva, ${ }^{1}$ Trine Jorgensen. ${ }^{1}$ Cleveland Clinic Lerner College of Medicine; ${ }^{2}$ Case Western Reserve University

\subsection{6/lupus-2019-Ism.107}

Background Neuropsychiatric lupus (NPSLE) is a common but poorly understood manifestation of systemic lupus erythematosus (SLE), affecting up to $80 \%$ of SLE patients. Current treatments include broad immunosuppression and symptomatic control rather than addressing pathogenesis. Current mouse models of NPSLE were developed by selective inbreeding until a complex genetic background resulted in spontaneous development of SLE. However, these models are not easily manipulated by genetic tools, which prevents elucidation of a more specific mechanism. We propose to establish the neurobehavioral phenotype of the B6.Nba2 spontaneous mouse model of SLE, known to depend on interferon (IFN)-. Interestingly, recent studies suggested that IFN- contributes to NPSLE by stimulating microglia, making it a promising potential drug target.

Methods Age- and sex-matched B6.Nba2 and B6 mice were tested $(n=3-5)$. We used open field testing as a general measure of movement and anxiety-like behavior and elevated plus maze as a more specific measure of anxiety. A novel object placement test assessed spatial memory. Rotarod testing was used to characterize motor coordination, and the forced swim test assessed for depression-like behavior. Students t-test with Welchs correction was used for statistical analysis.

Results B6.Nba2 mice moved less distance and at a slower velocity and spent less time in the center of the open field than B6 mice $(p=0.0005)$. To determine whether this was truly from anxiety or if it was a result of motor deficits, we ran elevated plus maze and rotarod tests. In the elevated plus maze, B6.Nba2 mice spent more time in the closed arms than 